\title{
Current clinical approach to patients with disorders of consciousness
}

\author{
Robson luis Oliveira de Amorim¹, Marcia Mitie Nagumo²*, Wellingson Silva Paiva ${ }^{3}$, Almir Ferreira de Andrade ${ }^{3}$, \\ Manoel Jacobsen Teixeira ${ }^{4}$ \\ ${ }^{1}$ PhD - Assistant Physician of the Neurosurgical Emergency Unit, Division of Neurosurgery, Hospital das Clínicas, Faculdade de Medicina, Universidade de São Paulo (FMUSP), São Paulo, SP, Brazil \\ ${ }^{2}$ Nurse - MSc Student at the Neurosurgical Emergency Unit, Division of Neurosurgery, Hospital das Clínicas, FMUSP, São Paulo, SP, Brazil \\ ${ }^{3}$ Habilitation (BR: Livre-docência) - Professor of the Neurosurgical Emergency Unit, Division of Neurosurgery, Hospital das Clínicas, FMUSP, São Paulo, SP, Brazi \\ ${ }^{4}$ Habilitation (BR: Livre-docência) - Full Professor of the Division of Neurosurgery, Hospital das Clínicas, FMUSP, São Paulo, SP, Brazil
}

\begin{tabular}{|c|c|}
\hline & SUMMARY \\
\hline $\begin{array}{l}\text { Study conducted at Hospital das Clínicas, } \\
\text { Faculdade de Medicina, Universidade de } \\
\text { São Paulo (FMUSP), São Paulo, SP, Brazil }\end{array}$ & $\begin{array}{l}\text { In clinical practice, hospital admission of patients with altered level of conscious- } \\
\text { ness, sleepy or in a non-responsive state is extremely common. This clinical con- } \\
\text { dition requires an effective investigation and early treatment. Performing a fo- }\end{array}$ \\
\hline $\begin{array}{r}\text { Article received: } 1 / 28 / 2015 \\
\text { Accepted for publication: } 5 / 4 / 2015\end{array}$ & $\begin{array}{l}\text { cused and objective evaluation is critical, with quality history taking and } \\
\text { physical examination capable to locate the lesion and define conducts. Imaging }\end{array}$ \\
\hline *Correspondence: & and laboratory exams have played an increasingly important role in supporting \\
\hline $\begin{array}{l}\text { Iress: Av. Dr. Enéas de Carvalho Aguiar, } \\
\text { 255, Cerqueira César }\end{array}$ & clinical research. In this review, the main types of changes in consciousness are \\
\hline São Paulo, SP - Brazil & discussed as well as the essential points that should be evaluated in the clinical \\
\hline $\begin{array}{l}\text { Postal code: } 05403-000 \\
\text { marcianagumo@gmail.com }\end{array}$ & management of these patients. \\
\hline
\end{tabular}

Keywords: coma, neurology, emergency medicine, state of consciousness.

\section{INTRODUCTION}

Consciousness is the individual's ability to have perception of themselves and their surrounding environment. It is important to distinguish the level and the content of consciousness. The first refers to the degree of alertness and the second is related to the quality and coherence of the individual's thought, cognition and attitude. To be conscious one must be awake; however, being awake does not necessarily mean that one is conscious. Anatomically and functionally, consciousness depends on the interaction and activity of the cerebral cortex, brainstem and thalamus. ${ }^{1}$ The activity of these three brain regions has fundamental importance on an individual's capacity to open their eyes, respond to verbal and motor commands and also be aware of themselves and the environment through the interrelation of the five senses (sight, smell, touch, taste, and hearing).

The most common causes of brain injury are traumatic, ischemic/hemorrhagic and metabolic (drugs, excess insulin, diabetes, alcohol) $)^{2}$ in nature.

Altered levels of conscience can range from temporospatial disorientation to a state of deep coma. ${ }^{3}$ Therefore, know how to correctly classify the patient's type of alteration is as important as defining appropriate medical conduct for the clinical symptoms.
The different stages that may precede coma will be described below. Drowsiness or lethargy is considered a state of decreased level of consciousness in which the patient can be woken up with mild stimuli and obey commands. In the state of torpor, the patient wakes up after mechanical stimulation, responds to simple requests, has self-psychic orientation, while generally suffering allopsychic disorientation. ${ }^{4}$ A patient with clouding of consciousness oscillates between states of drowsiness and agitation. Stupor is considered a deeper state of drowsiness in that the individual needs to receive vigorous and repeated stimuli to be awakened.

Coma can then be defined as a sustained state ( $>1$ hour) of loss of perception of oneself and of the surrounding environment, characterized by a reduction in the level of alertness to such an extent that one cannot be awakened after maximum internal or external stimulus. Classically, coma is considered in patients scoring less than nine points on the Glasgow Coma Scale (GCS). In a coma, the central nervous system is compromised, characterized by ascending reticular activator system injury (ARAS) and/or extensive injury to the cerebral hemispheres. This may be due to a structural injury or metabolic disorder, and information such as previous symptoms, his- 
tory of recent trauma, personal history, life habits and medications in use are valuable for clinical management. ${ }^{3}$

Among the states that are characterized by altered content of consciousness are delirium and dementia. Delirium is characterized by disorientation, attention deficit, a feeling of fear, irritability and changes in the perception of sensory stimuli, such as visual hallucinations. ${ }^{2}$ In dementia, there is the progressive and permanent loss of cognitive function over months or years, without changes in alertness or level of consciousness.

The following behavioral states can be commonly confused with coma: persistent vegetative state, minimally conscious state, abulia, catatonia, locked-in syndrome, akinetic mutism and psychogenic passivity (Table 1). The first two can be states subsequent to coma.

TABLE 1 Types of consciousness disorder; -(absent), +(present); UWS: unresponsive wakefulness syndrome.

\begin{tabular}{lll} 
State & Alert & Consciousness \\
\hline Coma & - & - \\
\hline Persistent vegetative state/UWS & +++ & - \\
\hline Minimally conscious state & +++ & + \\
\hline Delirium & ++ & ++ \\
\hline Abulia & ++ & ++ \\
\hline Catatonia & ++ & + \\
\hline Akinetic mutism & +++ & + \\
\hline Locked in syndrome & +++ & +++ \\
\hline Psychogenic passivity & +++ & +++ \\
\hline
\end{tabular}

The clinical definition of the vegetative state, most recently described as unresponsive wakefulness syndrome (UWS $)^{5,8,10}$ the clinical condition of complete unconsciousness of self and the environment. However, the sleep-wake cycle is maintained and the autonomic functions of the hypothalamus and brainstem are completely or partially preserved. ${ }^{6,8}$ The patient usually presents reflexes, eye opening and spontaneous breathing and may perform spontaneous movements like chewing and swallowing, emitting unintelligible sounds, and demonstrating certain spontaneous reactions such as smiling and crying. The main causes are TBI and cardiorespiratory arrest. These symptoms must be present for more than 4 weeks after the event that led to brain injury. ${ }^{6}$

In a minimally conscious state, the patient may possess some degree of consciousness, obey verbal commands sporadically, and try to communicate; however, this is most often unintelligible, and they may cry or smile in response to affective stimuli, as well as track moving objects, voices or people.
Abulia is a behavior in which there is serious indifference with a reduction or absence of emotional or mental behavior, in which the patient does not speak or move spontaneously, although alert and recognizing stimuli from the environment. ${ }^{3}$ This usually occurs in patients with bilateral frontal lesions. Akinetic mutism presents symptoms similar to those of abulia but less severe; the patient shows an unwillingness/extreme difficulty to move or speak, with the level and content of consciousness preserved, and eyes following the observer or sound although the patient does not obey commands. Muscle tone and reflexes are usually intact. In the case of locked-in syndrome, the patient also has the level and content of consciousness preserved; however, the patient presents complete paralysis, preventing any type of movement or verbal communication. In some cases, eye movement may be present. This syndrome usually occurs in patients with basilar artery thrombosis and ischemic infarction of the base of the pons and must be differentiated from coma and persistent vegetative state. This condition may show symptoms similar to those of acute polyneuropathies, myasthenia gravis and acute use of neuromuscular blockers. Advances in the field of neuroimaging suggest a new diagnosis: Functional locked-in syndrome. In this condition, patients do not show any behavioral signs of consciousness and differ from patients who are in a vegetative state as only examinations such as functional magnetic resonance imaging, positron emission tomography or evoked potential tests are able to identify responses that suggest some degree of consciousness. ${ }^{10}$ Catatonia is a state in which the individual may remain mute and with a marked decrease of motor activity, usually associated with psychiatric symptoms, but it can also occur due to metabolic disorders or induced by drugs. ${ }^{3}$ The patient exhibits bizarre and repetitive behavior, posture disturbances and rigidity.

Psychogenic passivity is also associated with psychiatric conditions and includes preserved muscular tone, resistance to passive movement of the limbs, resistance to opening of the eyelids or forcibly closed eye, eyes focused on the ground regardless of the side on which they are lying, or the presence of non-epileptic seizures.

So, how can we differentiate a patient in a coma from a brain dead patient? In brain death, brain damage is so extensive that there is no potential for structural and functional recovery of the brain and internal homeostasis cannot be maintained. What separates the state of coma from a diagnosis of brain death is the irreversible nature of the latter, with systemic repercussions on the homeostasis of vital organs, based on permanent focal or diffuse structural damage to the brain. 


\section{History and clinical examination}

The identification of the cause of the coma must be initiated by gathering information from family members and people who may have witnessed the clinical progression of the patient. It is very important to obtain information with respect to the start and progression of symptoms (sudden or gradual), life habits (possible use of drugs or toxic substances), presence of fever, history of trauma, previous symptoms or diseases, personal and psychiatric history and medication in use.

The general clinical examination should seek evidence of systemic conditions that could lead to changes in the level of consciousness. Vital signs and the examination of the cardiovascular, pulmonary and gastrointestinal systems, skin and other systems may provide important data regarding the etiology of the coma.

The approach should be carried out in a systematic manner in order for it to be concise and efficient: checking if the airways are patent; checking if breathing is providing adequate oxygenation (observing the respiratory pattern, oxygen saturation). Oxygen should be provided in cases of hypoxemia or establishing airway and ventilatory assistance if the GCS score is lower than nine points. Blood pressure should be measured, verifying the heart rate and obtaining intravenous access, as well as checking blood glucose to exclude the suspicion of hypoglycemia. Signs of trauma, evidence of recent epilepsy crises (tongue lesions, sphincter release) should be sought, evaluating the skin, ophthalmoscopy, evidence of exogenous poisoning (needle marks suggesting drug use), evaluation of the breath, presence of neck stiffness, and temperature assessment.

Fever is most common in systemic infections such as pneumonia, bacterial meningitis or viral encephalitis. An excessively high temperature is related to burns and poisonings by drugs with anticholinergic effects. Hypothermia can occur in intoxicated patients, those in shock, in barbiturate poisoning and myxedema.

An abnormally low pulse suggests a heart block by medications such as tricyclic antidepressants or anticonvulsants. Severe hypertension is observed in intracranial hemorrhage and hypertensive encephalopathy.

Cherry-red skin coloration is typical of carbon monoxide poisoning. Abrasions, epistaxis and otorrhagia, and hematoma in the cephalic region suggest a traumatic cause of coma. Hyperemia of the face and conjunctiva is a common finding in alcoholics. Maculopapular rash suggests meningococcemia, staphylococcal endocarditis and typhoid fever. Excessive sweating is found in hypoglycemic patients or those in shock. Excessively dry skin is found in diabetic ketoacidosis and uremia.
The simple assessment of breath odor can provide the diagnosis of the cause of the coma. The odor of alcohol is easily recognized and in patients with symptoms such as uremia, hepatic coma, diabetic ketoacidosis and cyanide poisoning, typical odors can be distinguished by an acute sense of smell.

\section{Neurological examination}

The most important data from the neurological examination for the location and prognosis include: level of consciousness, size and pupillary response to light, spontaneous or reflex ocular motility, skeletal motor response and breathing pattern.

\section{Level of consciousness}

The assessment of the level of consciousness should include a description of the patient's alertness, and response to verbal and painful stimuli. The goal is to determine the degree of alteration to the level of consciousness and have a clinical parameter for evolution and prognosis. It should be carried out serially and following similar criteria and standards among the examiners for comparative purposes. It should begin with verbal stimulus and in the absence of a response, followed by painful stimulus. The painful stimulus can be applied to the supraorbital region, nail bed or sternum. Asymmetric motor responses are suggestive of focal hemispheric damage.

The GCS is a standardized scale used to assess the level of consciousness originally designed for patients suffering traumatic injury, which, however, can also be used to assess any disturbance of consciousness in the acute phase.

\section{Pupil size and reactivity to light}

Pupil reactions have fundamental importance in the examination as well as the shape, size and symmetry presented by the pupils. A unilateral pupillary increase $(>5.5$ $\mathrm{mm}$ ) is an early indicator of compression or stretching of the oculomotor nerve (cranial nerve III) as a side effect of an expansive unilateral process. Initially, there is a decrease in reaction to unilateral light.

Pupil size and reactivity are dependent on the action of the sympathetic and parasympathetic neurons that innervate the pupil dilation and constriction muscles. The sympathetic system stimulates the contraction of the pupil dilation muscles, causing mydriasis, while the parasympathetic system stimulates pupil contraction muscles, leading to miosis. At rest, there is a continuous tonic action of both the sympathetic and parasympathetic systems. If there is impairment of the sympathetic or 
parasympathetic pathways, the effect on pupil size, miosis or mydriasis, will depend on the action of the system affected the least or still intact. The pupillary reflex consists of the pupillary contraction after light stimulation via the parasympathetic pathway.

As the areas related to consciousness control are adjacent to these pathways, pupil changes can help us in the differentiation and location of possible causes of coma.

Hypothalamic lesions, especially in the posterior and ventrolateral region, can produce ipsilateral pupillary constriction, usually associated with ptosis and anhidrosis (Horner syndrome). Thalamus lesions can lead to small, reactive pupils, known as diencephalic pupils.

Mesencephalic lesions can produce three types of pupil changes depending on the location of the damage: dorsal tectal region lesions, lesions that disrupt pupillary reaction to light, pupils with medium or little dilation (5 to $6 \mathrm{~mm}$ ), and fixed pupils with preservation of the accommodation reflex. Pupillary size oscillations may occur (hippus) alongside maintenance of the ciliospinal reflex; mesencephalic nuclear lesions, usually affect both the sympathetic and parasympathetic pathways, leading to medium-fixed pupils ( 4 to $5 \mathrm{~mm}$ ), generally a little irregular, and generally related to transtentorial herniation; lesions of bilateral cranial nerve III lead to paralytic mydriasis, usually associated with uncal herniation. Pontine lesions lead to extreme bilateral miosis ( $<1 \mathrm{~mm})$, with preservation of the pupillary light reflex, which is very hard to perceive. Pinpoint pupils due to pontine hemorrhages may result from sympathetic pathway lesions and irritation of parasympathetic pathways.

In patients in a coma related to toxic-metabolic changes, in general the pupils are isochoric and reactive to light, except in the terminal stage. The actions of drugs whether local or systemic can lead to pupil changes due to their effect on the sympathetic or parasympathetic pathways. Anticholinergic drugs such as atropine or scopolamine, can lead to pupillary dilation. Opiates (heroin and morphine) can lead to miosis, similar to pontine hemorrhage. Barbiturates may lead to fixed pupils. Data from the clinical history and other data from the neurological exam can help us in the differentiation of these cases. Post-ictal patients usually present mydriatic but reactive pupils.

\section{Ocular motility}

Ocular motility depends on the integrity of structures located in the brain, cerebellum and brainstem. Since we cannot evaluate voluntary ocular motility in unresponsive patients, we must evaluate the integrity of reflex pathways located in the brainstem. The absence of changes in ocular motility means that the region located between the vestibular nuclei, in the medulla oblongata-pons junction, up to the oculomotor nuclei in the midbrain are intact.

In coma patients, we must evaluate three aspects with respect to ocular motility: (1) primary gaze at rest, to assess the presence of deviations; (2) observe the presence of spontaneous eye movements and (3) test eye movements.

The presence of deviations in the rest position can provide us with evidence of cranial nerve palsies. Conjugate deviations of the lateral gaze may be the result of lesions from the cortex to the contralateral pontine reticular formation, which is common in strokes. Disconjugate deviations of the lateral gaze may reflect abducens and oculomotor nerve paralysis, or internuclear ophthalmoplegia. The presence of downward eye deviations can mean brainstem lesions, compression of the mesencephalic tectum, which may be present in hydrocephalus. Thalamic and subthalamic lesions can lead to conjugate deviation both upward and downward. Other conditions that can lead to upward deviation of the eyes are sleep, epileptic seizure, syncope, apnea in Cheyne-Stokes respiration, bleeding in the cerebellar vermis, ischemia or brainstem encephalitis. Skew deviation is a vertical misalignment of the eyes, usually corresponding to brainstem or cerebellar lesions.

The observation of spontaneous eye movements can offer important information. The presence of roving movements, consisting of slow and spontaneous conjugate movements of the lateral gaze, can indicate the integrity of the oculomotor pathways and their connections. The presence of nystagmus in comatose patients can be indicative of an irritative supratentorial focus. Ocular bobbing is characterized by fast conjugate movements downwards followed by a slow return to the primary position, occurring generally in brainstem lesions, especially in the pons.

The evaluation of reflex eye movements can be done through the oculo-cephalic maneuver or the caloric test (vestibular-ocular). The oculo-cephalic maneuver is carried out by vertical or lateral rotation of the head and observation of the eye movement. This test is not conducted in patients with suspicion of trauma, due to the possibility of cervical lesion. In patients where this reflex is preserved, the eyes move in unison and in the opposite direction to the movement of the head.

The caloric test consists of stimulation of the vestibular-ocular pathways using cold water (50 to $100 \mathrm{~mL}$ ) applied to the auditory canal. The ears are irrigated separately in an interval lasting a few minutes. The normal response consists of a deviation of the slow phase conju- 
gate gaze to the stimulated side and a fast corrective movement or nystagmus in the contralateral direction. Loss of the fast phase is observed in comatose patients. This is a good examination in cases of suspected psychogenic origin of the coma. Obviously, a brainstem injury may lead to absent response by interrupting this reflex.

Through these maneuvers we can observe the integrity of oculomotor pathways in the brainstem and clearly show the palsy of isolated cranial nerves. The absence of a bilateral reflex response can indicate the presence of extensive lesions in the brainstem.

\section{Motor response}

The motor examination of comatose patients is carried out initially through observation of posture at rest, the presence of spontaneous movement or response to verbal or painful stimuli. The motor response should be analyzed in comparison to the opposite side and after symmetrical stimulation, in the four limbs.

Head and eye deviation to one side and contralateral hemiplegia indicate supratentorial lesion, while deviation to the same side of the hemiplegia may indicate a brainstem lesion. External rotation of the lower limb may be indicative of hemiplegia or hip dislocation.

Decerebrate posturing consists of bilateral extension of the lower limbs and adduction and internal rotation of the shoulders and extension of the elbows and wrists. This generally means a bilateral mesencephalic and pons lesion but can appear in severe metabolic encephalopathies and supratentorial lesions involving the bilateral corticospinal tract. Decorticate posturing consists of bending the elbows and wrists, adduction of the shoulders and extension of the lower limbs. Although not a posture with a good topographic correlation, it usually indicates injury above the brainstem. These abnormal postures can be observed spontaneously or after painful stimuli, and their presence might suggest a brainstem herniation syndrome.

Other movements that can be observed are: tonicclonic seizures in epileptic seizures; myoclonus, observed frequently in post-anoxic encephalopathy and other metabolic comas; reflex responses, such as the triple flexion response of the lower limbs and the plantar reflex (Babinski sign).

\section{Respiratory pattern}

In comatose patients, the respiratory pattern can help us with the topographic diagnosis of the lesion. These may include: Cheyne-Stokes respiration (bilateral hemispheric lesions, severe heart failure), central neurogenic hyperventilation (brainstem lesions), apneusis (lesions in the dorsal-medial region of the lower half of the pons), Biot's respiration (lower pontine tegmental lesion) and ataxic respiration (generally damage to the medulla oblongata).

\section{LABORATORY/IMAGING EXAMS}

The requested exams should include blood tests, electrolytes and biochemistry (sodium, calcium, phosphorus, magnesium, chlorine, glucose), renal, hepatic and thyroid functions, coagulogram, and blood gas. In selected cases, quantify the serum level of certain drugs and toxicological screening.

If there is suspicion of poisoning, aspiration and analysis of gastric contents can contribute to the diagnosis. According to the availability of the service, the serum concentration of anticonvulsants, opioids, diazepinic agents, barbiturates, alcohol and a large number of toxic substances can be measured.

White blood cell count can identify neutrophilic leukocytosis, present in bacterial infections such as meningitis, where it is usually greater than $12,000 / \mathrm{mm}^{3}$.

In some cases thyroid hormones and serum cortisol may be requested, such as in suspected myxedema coma and Addisonian crisis, respectively. One must mind that water and sodium disorders such as hypo- or hypernatremia can be results of brain disease that will directly or indirectly affect the hypothalamic-pituitary axis.

\section{Head tomography}

This is essential upon suspicion of intracranial lesion and must be the first image exam requested. It is performed in a few seconds, is available in most emergency services and has good sensitivity to detect bleeding (subarachnoid hemorrhage, subdural hematoma, epidural hematoma, or intraparenchymal hematoma), hydrocephalus, tumors and extensive brain infarcts. ${ }^{6}$

Sometimes it is difficult to determine if the abnormality shown in the head CT scan is responsible for the comatose state of the patient. In lesions that exert an acute expansive effect, measuring the deviation from the midline structures is an important parameter. Horizontal deviations of 3 to $5 \mathrm{~mm}$ cause drowsiness; from 5 to $8 \mathrm{~mm}$, they cause stupor; and greater than $9 \mathrm{~mm}$, they cause a coma. Patients with deviations exceeding $10 \mathrm{~mm}$ and who are still in a coma, probably present lesions growing from weeks to months (e.g. chronic subdural hematoma, brain tumor). On suspicion of meningitis, carry out head tomography whenever possible prior to performing lumbar puncture, since herniation of the brain- 
stem is a real possibility in the presence of intracranial hypertension. Structural causes of coma may be related to subtle changes or even a normal CT scan (e.g. unilateral chronic isodense subdural hematomas, subarachnoid hemorrhage, deep vein thrombosis, herpes simplex encephalitis, and meningitis).

\section{Cerebrospinal fluid}

Lumbar puncture is carried out upon suspicion of infection of the central nervous system or on suspicion of subarachnoid hemorrhage with a normal head CT scan.

\section{Magnetic resonance imaging}

This is not an examination carried out routinely in the emergency department. However, it may provide valuable information on the number, characteristics and severity of the lesions, ${ }^{10}$ as well as allow the identification of injuries not visible to the head CT, as in cases of herpes simplex encephalitis or diffuse axonal injury. ${ }^{7}$ Functional magnetic resonance imaging and positron emission tomography (PET-SCAN) have proved promising and can help in the differential diagnosis of patients in a minimally conscious or vegetative state. ${ }^{9}$

The latest technologies include diffusion-weighted imaging (DWI) and diffusion-tensor imaging (DTI), which are tests using methods similar to MRI, but with a more advanced technology. They provide in vivo images of biological tissues, and allow the observation of molecular water displacement as well as more accurate data about damage occurring in specific structures of the nervous system. ${ }^{10}$

\section{Electroencephalogram (EEG)}

This is most recommended upon suspicion of non-convulsive seizures, especially in patients with worsening of the level of consciousness that have a known underlying neurological disease. Some studies have shown promising results in the integration of transcranial magnetic stimulation (TMS) with simultaneous EEG for assessment of brain connectivity and cortical interactions. ${ }^{11,12}$

\section{Treatment of COMATOSE Patients}

The initial care of a comatose patient, the most serious altered state of consciousness, should emphasize basic precautions to maintain the airways and adequate ventilation, hemodynamic stability and other measures to minimize damage to the brain and other vital organs. We should exclude causes requiring urgent surgical intervention and medical causes that need immediate treatment.

In general, the patient has no ability to protect the airways and the presence of hypoxia or hypoventilation requires endotracheal intubation. At the same time, blood is collected for determination of glucose, electrolytes and renal and liver function tests. In patients with hypoglycemia, infusion of 25 to $50 \mathrm{~mL}$ of $50 \%$ glucose should always be supplemented with thiamine.

Gastric aspiration and lavage with saline solution can be a diagnostic and therapeutic measure upon suspicion of coma due to ingestion of drugs. Salicylates, opiates and anticholinergic drugs induce gastric atony and can be removed several hours after ingestion. Caustic materials should not be washed out because of the risk of gastrointestinal perforation. The administration of activated charcoal is indicated in certain cases of poisoning. This is not effective in poisoning caused by heavy metals, cyanides and alcohol.

In patients with intracranial hypertension that are getting worse neurologically (worsening of the GCS score, anisocoria, decerebrate or decorticate posturing), proceed with rapid infusion of $20 \% 1 \mathrm{~g} / \mathrm{kg}$ mannitol or hypertonic saline solution $(\mathrm{NaCl} 20 \% 1 \mathrm{~mL} / \mathrm{kg})$, associated with hyperventilation in order to maintain $\mathrm{pCO}_{2}$ at around 30 $\mathrm{mmHg}$. In lesions with expansive effects suggestive of brain tumors, a bolus of $20 \mathrm{mg}$ dexamethasone may be given. In selected cases, intracranial pressure monitoring may be appropriate. Lumbar puncture should be performed upon suspicion of meningitis and preferably after a CT scan of the head.

Extreme hyperthermia or hypothermia must be avoided. To prevent bladder distension, a catheter should be inserted. Aspiration pneumonia is prevented by proper positioning, restriction of oral fluids, gastric tube and orotracheal tube. As comatose patients have an increased risk of deep vein thrombosis, elastic compression stockings in the lower limbs and subcutaneous application of unfractionated heparin $5000 \mathrm{u}$ every eight hours are to be used (Figure 1).

\section{Conclusion}

The care of patients presenting altered state of consciousness is a medical emergency that requires a multidisciplinary team prepared to act quickly. Altered states of consciousness may have structural or metabolic causes, and clinical manifestations can include changes to pupillary reactivity, motor and verbal functions, vital signs, laboratory or imaging tests. Patient management begins with the assessment of airways, breathing pattern, hemodynamic support, monitoring of vital signs and systematic physical and neurological examination. Meanwhile, medical information should be gathered from family members, friends or the rescue team, searching for data 


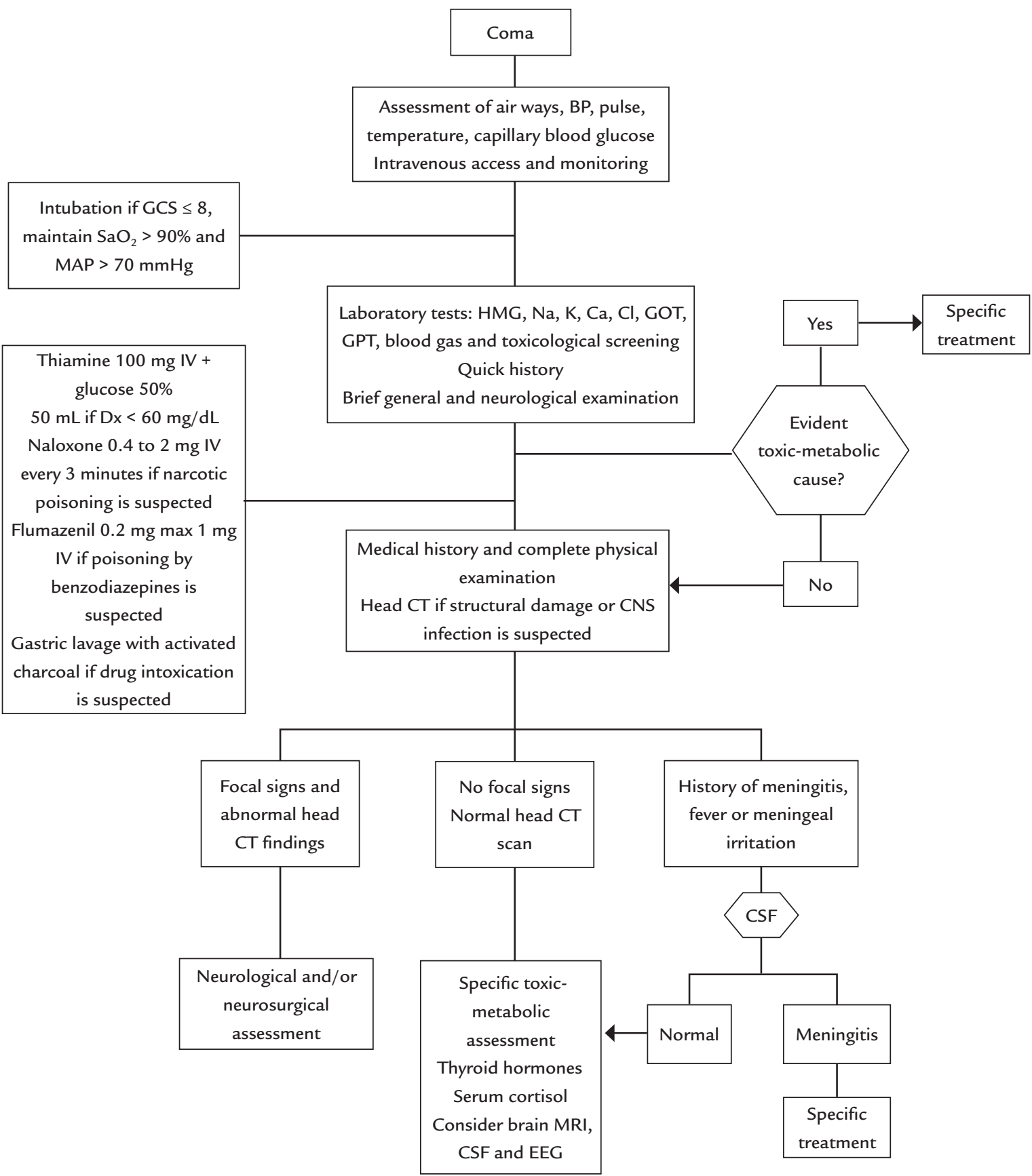

FIGURE 1 Algorithm of the initial approach to the patient in a coma.

BP: blood pressure; GCS: Glasgow Coma Scale; MAP: mean arterial pressure; HMG-CoA: 3-hydroxy-3-methyl-glutaryl coenzyme A; GOT: glutamic-oxaloacetic transaminase; GPT: glutamate-pyruvate transaminase; CT: computed tomography; CNS: central nervous system; CSF: cerebrospinal fluid; MRI: magnetic resonance imaging; EEG: electroencephalogram.

that could be essential for localization and etiology of the patient's symptoms. Basic laboratory investigations and imaging tests are of utmost importance for differential diagnosis.

The prognosis is extremely variable and depends on the cause, duration and extent of the affected site. However, effective initial care and introduction of proper treat- ment are crucial in order to prevent the occurrence of secondary damage and to speed up patient recovery.

\section{Resumo}

Abordagem clínica atual do paciente com distúrbios de consciência 
Na prática clínica é extremamente comum a admissão hospitalar de pacientes com nível de consciência alterado, sonolentos ou em estado não responsivo. Essa condição clínica demanda uma investigação eficaz e um tratamento precoce. É fundamental a realização de uma avaliação focada e objetiva, com a realização de anamnese e exame físico de qualidade para localizar a lesão e definir condutas. Exames de imagem e laboratoriais têm desempenhado papéis cada vez mais relevantes no suporte à investigação clínica. Nesta revisão, são discutidos os principais tipos de alterações de consciência e os pontos imprescindíveis que devem ser avaliados na abordagem clínica desses pacientes.

Palavras-chave: coma, neurologia, emergências, estado de consciência.

\section{RefEREnCES}

1. Gosseries O, Vanhaudenhuyse A, Bruno MA, Demertzi A, Schnakers C, Boly $\mathrm{MM}$, et al. Disorders of consciousness: coma, vegetative and minimally conscious states. In: Cvetkovic D, Cosic I (eds.). States of consciousness Experimental insights into meditation, waking, sleeping and dreams. The Frontiers Collection. Berlin: Springer, 2011. p.29-55.
2. Bonsignore LT, Macrì S, Orsi P, Chiarotti F, Alleva E. Coma and vegetative states: state of the art and proposal of a novel approach combining existing coma scales. Ann Ist Super Sanita. 2014; 50(3):241-8.

3. Andrade AF, Carvalho RC, Amorim RLO, Paiva WS, Figueiredo EG, Teixeira MJ. Coma e outros estados de consciência. Rev Med (São Paulo). 2007; 86(3):123-31.

4. Shinosaki JSM, Baiense RF. Manual de Neurologia. Manual do Residente da Universidade Federal de São Paulo - UNIFESP. São Paulo: Roca, 2009 .

5. Von Wild K, Laureys ST, Gerstenbrand F, Dolce G, Onose G. The vegetative state - a syndrome in search of a name. J Med Life. 2012; 5(1):3-15.

6. Practice parameters: assessment and management of patients in the persistent vegetative state (summary statement). The Quality Standards Subcommittee of the American Academy of Neurology. Neurology. 1995; 45(5):1015-8.

7. Moore SA, Wijdicks EF. The acutely comatose patient: clinical approach and diagnosis. Semin Neurol. 2013; 33(2):110-20.

8. Laureys S, Celesia GG, Cohadon F, Lavrijsen J, León-Carrión J, Sannita WG et al.; European Task Force on Disorders of Consciousness. Unresponsive wakefulness syndrome: a new name for the vegetative state or apallic syndrome. BMC Med. 2010; 8:68.

9. Stender J, Gosseries O, Bruno MA, Charland-Verville V, Vanhaudenhuyse A, Demertzi A, et al. Diagnostic precision of PET imaging and functional MRI in disorders of consciousness: a clinical validation study. Lancet. 2014; 384(9942):514-22.

10. Bodart O, Laureys S, Grosseries O. Coma and disorders of consciousness: scientific advances and practical considerations for clinicians. Semin Neurol. 2013; 33(2):83-90.

11. Di Perri C, Thibaut A, Heine L, Soddu A, Demertzi A, Laureys S. Measuring consciousness in coma and related states. World J Radiol. 2014; 6(8):589-97.

12. Ilmoniemi, RJ, Kicic D. Methodology for combined TMS and EEG. Brain Topogr. 2010; 22(4):233-48. 